REVISTA DE ESTUDIOS E INVESTIGACIÓN

EN PSICOLOGÍA Y EDUCACIÓN

(c) (†) ()

ISSN: 1138-1663; eISSN: 2386-7418

UDC / UMinho

2021, Vol. 8, No. 2, 305-311.

DOI: https://doi.org/10.17979/reipe.2021.8.1.8744

\title{
Reseña: MITCA Método de implementación de tareas para casa + Informe sobre los deberes escolares
}

\section{Review of: MITCA Homework implementation method +} Report on homework

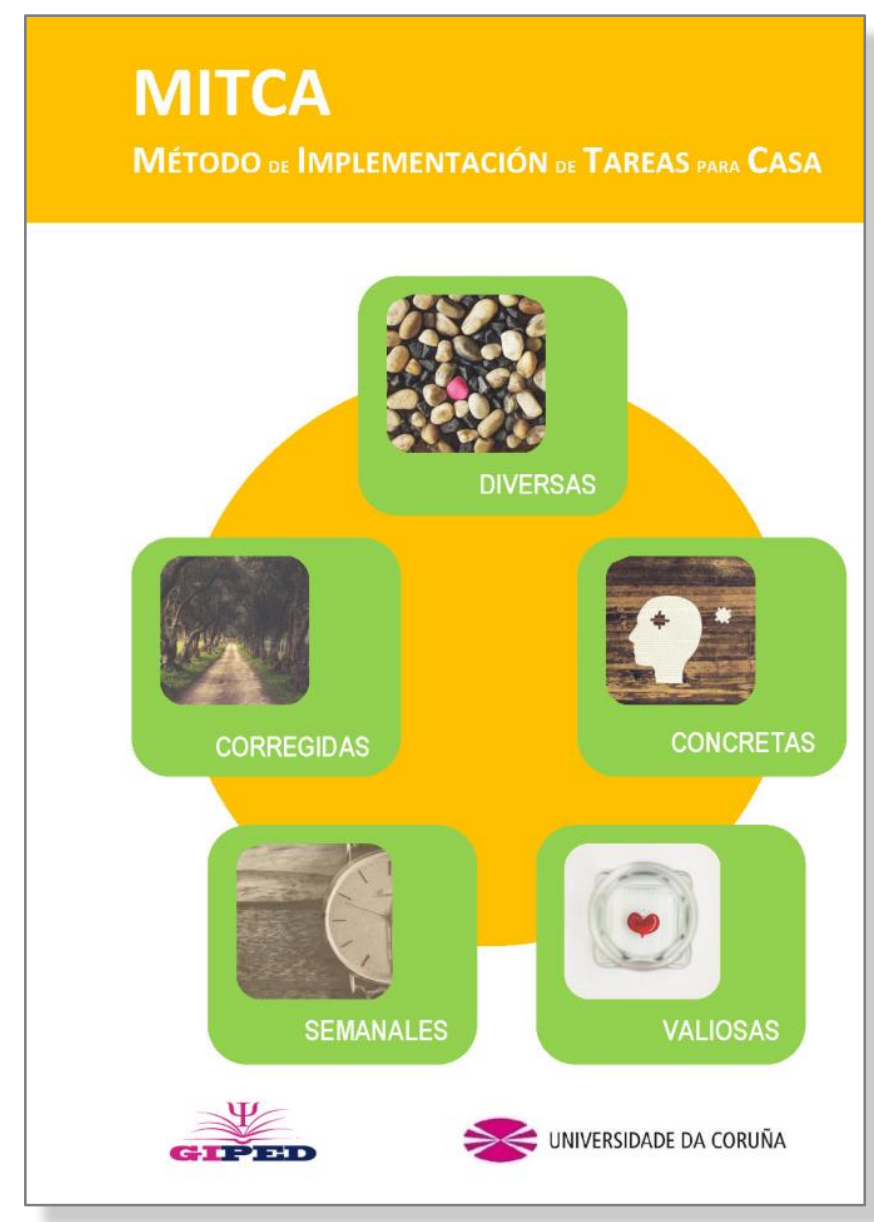

Antonio Valle-Arias

Susana Rodríguez-Martínez

(Coords.)*

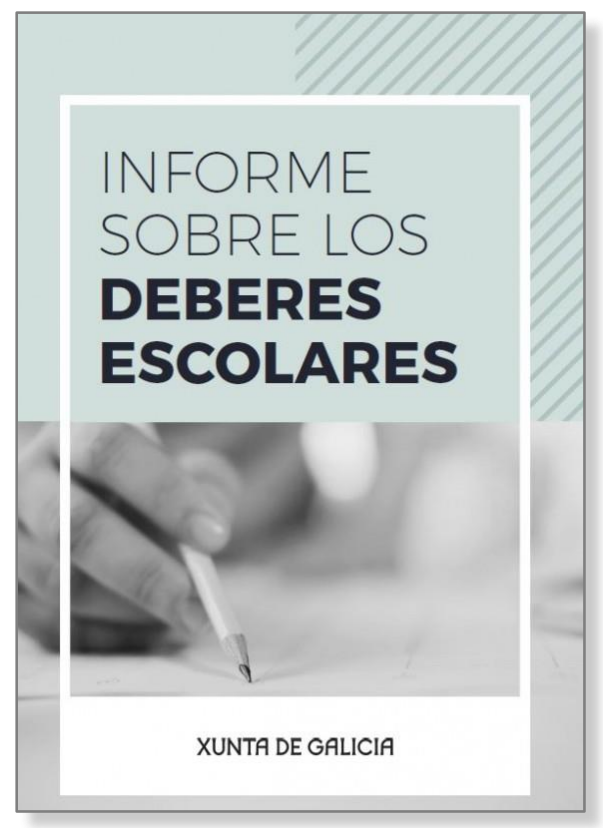

Antonio Valle-Arias

José Carlos Núñez-Pérez,

Pedro Rosário

(Coords.)*
* Más datos de ambas publicaciones en la última página. 
La Universidade da Coruña publicó recientemente un texto-manual, que es objeto de recensión en esta Revista, con la finalidad de dar a conocer y hacer más visible, en especial para los profesionales de la educación de niveles de enseñanza primaria y de secundaria, un modelo y método de ayuda al estudio del alumndo que realiza tareas para casa, los deberes escolares, de manera que sirva de ayuda, apoyo y refuerzo para el aprendizaje: es el MITCA, en cuanto "Método de implementación de tareas para casa". Previamente, una investigación financiada por la Xunta de Galicia, ha servido de base y fundamento científico y teórico-conceptual para este Método.

La tradición que se ha vivido y se vive en España ante el hecho de los deberes y tareas que el alumnado deberá realizar fuera del horario escolar ha estado muy arraigada en la sociedad desde hace varias décadas. Ha sido, y sigue siendo, un fenómeno controvertido, sujeto a discusión y debate, tanto desde la perspectiva de las familias, como implicadas que están en este tema, pero también por parte del profesorado y direcciones de los centros educativos en los niveles, prioritariamente de enseñanza primaria y secundaria.

Realizar tareas escolares en casa, como complemento y refuerzo al aprendizaje, supone de hecho un tiempo extra dedicado a la escuela, aunque no sea propiamente dentro de las aulas. Quizás también por eso, con frecuencia, se valora y evalúa si los niños pasan demasiadas horas pendientes de una planificación que puede ser demasiado rígida y con un abultado horario de dedicación al estudio.

Dos publicaciones de la autoría del Equipo dirigido por Antonio Valle Arias, catedrático de Universidad de Psicología de la Educación e integrado, entre otras, por las profesoras Susana Rodriguez, Isabel Piñeiro, Bibiana Regueiro, Iris Estévez, José Carlos Núñez y Natalia Suárez, han visto la luz en Galicia recientemente: una editada por la Xunta de Galicia y la otra por la Universidade da Coruña. Estas dos publicaciones están estrechamente relacionadas y, es por eso, que haré una recensión conjunta.

Informe sobre los deberes escolares es el título de la primera publicación, en formato libro de 85 páginas, editada por la Consellería de Cultura, Educación y Ordenación Universitaria (Xunta de Galicia). Está coordinado este libro por los profesores Antonio Valle de la Universidade da Coruña, José Carlos Nuñez, de la Universidad de Oviedo y Pedro Rosario de la Universidade do Minho. Surge esta obra como consecuencia de un proyecto concedido al Grupo de Investigación en Psicoloxía Educativa (GIPED), dirigido por el profesor Antonio Valle Arias y a la Red de Investigación RIES (Rede de Inmigración, Educación e Sociedade). Aparte de estar 
elaborado el libro por este equipo de investigadores -de un alto prestigio en Galicia y en España-, posee además otras connotaciones importantes que hay que destacar. En primer lugar, se refiere al tema de investigación: los deberes escolares. Se decía al principio que es éste un tema controvertido, sujeto a debate y discusión por diferentes sectores de la comunidad educativa y de la sociedad. Pues bien, en segundo lugar, de la otra connotación hay que decir ya que el debate y la discusión se hace ahora como consecuencia de un proceso de investigación, que es complejo, que exige mucha reflexión precisamente sobre los datos, sobre evidencias empíricas y con criterios científicos. Y aquí radica, a mi juicio, lo más importante de esta obra: su elaboración científica. Hay un tercer aspecto complementario y que es su aportación muy clarificadora para la comunidad educativa y, en general para la sociedad, respecto de la situación, importancia y funcionalidad de los deberes escolares dentro de la situación educativa de Galicia, aunque también, quizás, extrapolable al contexto español.

Todo el libro gira en torno a tres temas centrales: uno, al contexto y la justificación del abordaje de los deberes escolares, otro, a las variables implicadas en la realización de los deberes escolares y, finalmente, a las prudentes y cuidadosas conclusiones que se aportan y a sus aplicaciones prácticas.

En el primer tema los autores se hacen varias preguntas de total interés y trascendencia: ¿son los deberes que se prescriben para casa de calidad y se ajustan a las necesidades particulares de los niños? ¿son significativos...? ¿ ¿sirven para implicar más a los y las estudiantes y vincular a sus familias? Estas preguntas de hecho llevan a un análisis y debate que se hace de una manera muy detallada, pormenorizada, destacando los argumentos a favor y en contra de los deberes escolares.

En el segundo apartado del libro se plantean cuáles son las variables que están implicadas en la realización de los deberes escolares. Y aquí destacan varias que, por su importancia en el quehacer diario del profesorado y alumnado de primaria y secundaria, poseen un alto interés educativo. Refieren los autores la existencia de tres grupos de variables que están presentes en la realización de las tareas para casa.

Un primer grupo de variables hace referencia inevitablemente al rendimiento académico y los deberes escolares. Se aportan aquí varias cuestiones de indudable interés: se dice que los niños más pequeños tienen hábitos de estudio menos efectivos con lo cual se disminuye el efecto de los deberes en el rendimiento, pero, además se apunta que los profesores de educación secundaria suelen asignar menos deberes con el fin de mejorar las habilidades de gestión del tiempo y, así, centrarse más en la materia objeto de examen. En otro trabajo 
realizado por los autores de este libro, con una muestra de alumnado de educación primaria, se demuestra que quienes tienen mayor rendimiento son los que están más motivados intrínsecamente y más interesados en hacer los deberes... por tanto, se concluye que hacer los deberes implica que el rendimiento académico mejora cuando se hacen las tareas para casa.

Un segundo grupo importante de variables que explican las tareas se relacionan con la implicación de los y las estudiantes en sus propios deberes escolares. Se hace un análisis muy detallado de los datos y resultados recogidos durante el trabajo de investigación y, así, se aborda el tema de la cantidad de deberes realizados de los prescritos por el profesorado y, aquí, se ha demostrado que cuanto más motivados están hacia los deberes, más aprovechan el tiempo, gestionan mejor su motivación, presentan menos ansiedad y realizan mayor cantidad de deberes. Se hace mención también en este apartado al papel de las variables motivacionales y cognitivas. En este punto se alude a los trabajos de autoría del equipo de este libro, por ejemplo, se sabe que la implicación motivacional en los deberes disminuye a medida que transcurre en el tiempo la escolaridad del alumnado, de ahí que resulta necesario conocer si entiende el propósito de los deberes escolares y si valora hacerlos y, además, si comprende cómo los deberes pueden afectar a su rendimiento.

Por último, apuntan los autores que el tercer grupo de variables que intervienen en los deberes escolares provienen del profesorado y de la familia. Del profesorado, se afirma, porque es importante el propósito que se persigue con las tareas, porque es siempre fundamental su corrección y el feedback que produce con sus variantes y, por otra parte, la implicación de los padres parece que tiene efectos relevantes como promoción de la autonomía, el control e incentivo del aprendizaje que, a su vez, incrementa aspectos clave como son la mejora de las estrategias de estudio, colaboración y autorregulación, así como control de las emociones.

Finaliza este Informe sobre os deberes escolares con una serie de conclusiones y aplicaciones prácticas que incluyen las 14 últimas páginas del libro. Entre ellas, que a cualquier edad es siempre mejor hacer los deberes que no hacerlos; dedicarle más tiempo no siempre es lo mejor, sino que es fundamental su aprovechamiento. Se aconseja que sean siempre claros de manera que el alumnado vea su utilidad, que las tareas que se proponen sean atractivas y de calidad, que se dé siempre un feedback que busque el éxito y que implique una dosis de motivación para que se aprecie que el esfuerzo siempre está detrás del éxito.

Como ya se comenta al inicio de esta recensión, puede considerarse este Informe sobre os sobre os deberes escolares, coordinado por el Equipo del profesor Antonio Valle, como una primera parte de lo que resulta ser una segunda parte: el MITCA Método de Implementación de 
Tareas para Casa. Es muy oportuno el MITCA precisamente porque, a mi juicio, es el complemento, la respuesta adecuada con la que se pueden comprender mejor aquellas actividades escolares de ayuda y refuerzo para el aprendizaje, al menos con una función clara: la de ayudar a la autorregulación del aprendizje y a la búsqueda del compromiso escolar de los etudiantes. Los autores están buscando con MITCA que las actividades que se programen para casa sean entendidas por el alumnado como interesantes y valiosas para su avance académico, que tengan una finalidad clara y sean sensibles a la diversidad, que le sirvan para autoevaluarse y que contribuyan a su mejora y a la planificación de su tiempo.

EI MITCA, como método de ayuda al estudio y aprendizaje se describe en tres fases: preparación, trabajo y reflexión. En la preparación de las tareas para casa se atiende a tres procesos básicos, como la definición de la tarea, fijación de objetivos y planificación de la actividad. En la fase de trabajo para casa se precisa una mayor autorregulación y, por eso, con este método lo que se persigue es apoyar la mejor distribución y aprovechamiento del tiempo por parte del alumnado. Finalmente está la reflexión y, aquí, se pretende la búsqueda de una retroalimentación docente y que se convierta en un instrumento que facilite la comparación de los resultados de las tareas realizadas en casa con las tareas y actividades del aula de manera que, con ello, se pretende una revaluación semanal del trabajo realizado. Por eso se aclara que, con esta fase de reflexión se pretende que los y las estudiantes se auto-examinen para comprobar lo que saben y, sobre todo, aquello que todavía no dominan, pudiendo mejorar su rendimiento.

Hay algo importante a partir de aquí, ya que los autores le conceden especial relevancia, y es la caracterización de las tareas a realizar por el alumnado. Se informa y se justifican cuáles y qué elementos deben conllevar las tareas para casa: serán diversas, serán concretas, valiosas, serán semanales y serán corregidas. Con el método MITCA se explica cada una de las citadas tareas con sus pasos a seguir y, sobre todo, se expone el fundamento psicológico y educativo de cada una de ellas. Por ejemplo, cuando se afirma que las tareas deben ser valiosas lo que se hace es instar al profesorado a transmitir la utilidad, interés e importancia de la tarea al alumnado. La explicación que se da es que las tareas, cuando se prescriben, pueden mejorar su eficacia si se clarifican las expectativas, se ajusta, si es posible, su interés intrínseco y se identifica su valor instrumental; por eso considero que los autores del MITCA consiguen la finalidad de lo que buscan cuando se define la caraterística de tarea valiosa: aquella que tiene un valor intrínseco, con un valor de logro y con un valor de utilidad. Y así se van delimitando las características que reúne la prescripción de las tareas para casa. 
En definitiva, me parece MITCA un método excelente para optimizar el estudio, un modelo de refuerzo y de apoyo a las tareas del alumnado. También, y especialmente, es un método-guía, y a su vez de apoyo y estímulo, para el profesorado ya que se le informa sobre cómo se deben prescribir las tareas, su variedad, su concreción, sus correcciones y sobre todo su eficacia. A mi juicio, destacaría la importancia que para los autores de MITCA supone una óptima fundamentación de las tareas para casa en la psicología educativa, en una línea y enfoque cognitivo-conductual, y siempre teniendo presente los últimos e importantes hallazgos que se están operando en la actualidad en lo referente a las estrategias de aprendizaje y autorregulación del estudio, así como en los procesos cognitivos y motivacionales para el contexto educativo.

Alfonso Barca-Lozano (D) https://orcid.org/0000-0002-0618-8273 Facultade de Ciencias da Educación, Universidade da Coruña A Coruña - España barca@udc.es

Fecha de recepción: 24 de octubre de 2021. Fecha de revisión: 24 de noviembre de 2021. Fecha de aceptación: 25 de noviembre de 2021. Fecha de publicación: 1 de diciembre de 2021. 


\title{
DATOS DE LAS PUBLICACIONES RESEÑADAS
}

\author{
Antonio Valle Arias, Susana Rodriguez Martínez (Coords.) \\ Universidade da Coruña, España \\ MITCA: Método de implementación de tarefas para a casa \\ A Coruña, 2020 \\ Universidade da Coruña, Servizo de Publicacións \\ Núm. de páxinas: 52 \\ ISBN: 978-84-9749-799-2 \\ Depósito legal: C 278-2021 \\ https://doi.org/10.17979/spudc.9788497497992 \\ https://ruc.udc.es/dspace/handle/2183/27393 \\ MITCA Método de implementación de tareas para casa \\ A Coruña, 2020 \\ Universidade da Coruña, Servizo de Publicacións \\ Núm. de páginas: 52 \\ ISBN: 978-84-9749-793-0 \\ Depósito legal: C-1548-2020 \\ https://doi.org/10.17979/spudc.9788497497930 \\ https://ruc.udc.es/dspace/handle/2183/26698 \\ MITCA: Homework implementation method \\ A Coruña, 2020 \\ University of A Coruña, Publication Service \\ Number of pages: 47 \\ ISBN: 9788497496360 \\ Legal Deposit: C 108-2021 \\ https://doi.org/10.17979/spudc.9788497497930 \\ https://ruc.udc.es/dspace/handle/2183/27308
}

\author{
Antonio Valle-Arias ${ }^{1}$, José Carlos Núñez-Pérez ${ }^{2}$, Pedro Rosario ${ }^{3}$ (Coords.) \\ ${ }^{1}$ Universidade da Coruña, ${ }^{2}$ Universidad de Oviedo, ${ }^{3}$ Universidade do Minho \\ Informe sobre os deberes escolares \\ Informe sobre los deberes escolares \\ Santiago de Compostela, 2017 \\ Consellería de Cultura, Educación e Ordenación Universitaria, Xunta de Galicia. \\ Número de páginas: 85 \\ Depósito legal: C 1599-2017.
}

